\title{
Morphology and dynamics of aurora at fine scale: first results from the ASK instrument
}

\author{
H. Dahlgren ${ }^{1}$, N. Ivchenko ${ }^{1}$, J. Sullivan ${ }^{2}$, B. S. Lanchester ${ }^{2}$, G. Marklund ${ }^{1}$, and D. Whiter ${ }^{2}$ \\ ${ }^{1}$ Space and Plasma Physics, School of electrical engineering, KTH, Stockholm, Sweden \\ ${ }^{2}$ School of Physics and Astronomy, University of Southampton, UK
}

Received: 12 December 2006 - Revised: 11 April 2007 - Accepted: 11 April 2007 - Published: 28 May 2008

\begin{abstract}
The ASK instrument (Auroral Structure and Kinetics) is a narrow field auroral imager, providing simultaneous images of aurora in three different spectral bands at multiple frames per second resolution. The three emission species studied are $\mathrm{O}_{2}^{+}(5620 \AA), \mathrm{O}^{+}(7319 \AA)$ and $\mathrm{O}$ (7774 $\AA$ ). ASK was installed and operated for the first time in an observational campaign on Svalbard, from December 2005 to March 2006. The measurements were supported by data from the Spectrographic Imaging Facility (SIF). The relation between the morphology and dynamics of the visible aurora and its spectral characteristics is studied for selected events from this period. In these events it is found that dynamic aurora is coupled to high energy electron precipitation. By studying the $\mathrm{O}_{2}^{+} / \mathrm{O}$ intensity ratio we find that some auroral filaments are caused by higher energy precipitation within regions of lower energy precipitation, whereas other filaments are the result of a higher particle flux compared to the surroundings.
\end{abstract}

Keywords. Ionosphere (Auroral ionosphere; Particle precipitation; Instruments and techniques)

\section{Introduction}

The energy distributions of precipitating electrons within dynamic small scale structures of the aurora provide the key to understanding the acceleration mechanisms that are operating above the ionosphere. Information about these energies and their variation in space and time can be obtained from the spectral signatures of the auroral emissions. Simultaneous imaging in different wavelength regions at high time resolution can be used to investigate the relationship between incoming electron energy flux and spatial structure within auroral forms (Semeter et al., 2001).

Correspondence to: $\mathrm{H}$. Dahlgren

(hanna.dahlgren@ee.kth.se)
One method for obtaining estimates of the energies of precipitating electrons is from the ratios of emissions in different spectral lines (Kaila, 1989; Semeter, 2003, and references therein). Ivchenko et al. (2004) used imaging, spectral and incoherent scatter data to show that the presence of low energy electron precipitation results in an enhancement in the $\mathrm{O}^{+}\left({ }^{4} \mathrm{P}-{ }^{4} \mathrm{D}^{0}\right)$ multiplet compared to the $\mathrm{N}_{2}^{+} 1 \mathrm{~N}(0,2)$ bands. This $\mathrm{O}^{+}$enhancement was also shown to be linked with the presence of rayed arcs. Ivchenko et al. (2005) presented a study in which an auroral curl was shown to lack low energy precipitation, while rayed structures contained both high and low energy precipitating flux. These studies strongly suggest that there can be physical differences in the generation mechanisms resulting in auroral curls and rays. This is a different interpretation from that of earlier studies which explained rays as curls or folds seen from the side (Paschmann et al., 2003).

It has been suggested that curls are the result of an electrostatic instability within the electron acceleration region (Wagner et al., 1983; Vogt et al., 1999), while finescale structure is linked to highly energy-dispersed electron bursts accelerated by inertial Alfvén waves (Stasiewicz et al., 2000). This interpretation is supported by a study by Hallinan et al. (2001), which combined optical and in situ rocket measurements to show that Alfvén waves appeared to be accelerated electrons dispersively in the region above tall auroral rays. The waves were also present above a curled arc, but here they appeared more of a passive indicator of a KelvinHelmholtz instability in the large-scale acceleration region and were not directly affecting the incoming particles. Semeter and Blixt (2006), on the other hand, attempted to explain the time development of multiple narrow auroral filaments in terms of Alfvén wave dispersion. A narrow arc filament $(<100 \mathrm{~m})$ reported by Lanchester et al. (1997) had curl-like structures moving along its length. Optical and radar data were used as input to modelling to estimate the variation of energy flux across this structure. In this case the narrow core

Published by Copernicus Publications on behalf of the European Geosciences Union. 


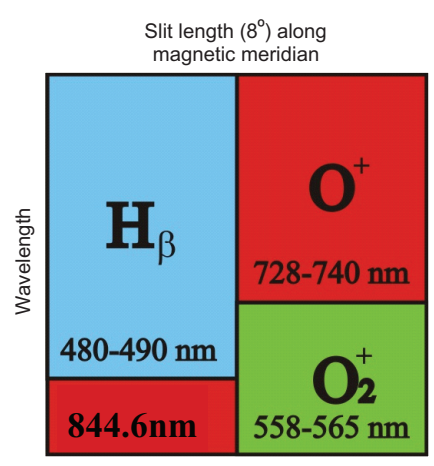

Fig. 1. Filter mosaic of the SIF spectrograph. Marked on each filter is the wavelength range the filter transmits and the main auroral emission species in this interval.

of the optical arc was found to contain a large energy flux of monoenergetic electrons embedded in a broader spectral distribution. The thin optical filament and its surrounding background were modelled using a 3-D multifluid simulation (Otto and Birk, 1993) which views the acceleration process as a decoupling between the magnetosphere and ionosphere, resulting in a highly time-dependent multiscale process.

In this paper we present data from a new multi-spectral imager, the ASK (Auroral Structure and Kinetics) instrument, combined with data from the Spectrographic Imaging Facility (SIF). Using these new measurements from selected spectral regions, we examine the connection between narrow auroral arc filaments and their spectral characteristics, and aim to further our understanding of the role of various acceleration processes in the production of small scale and dynamic structure in the aurora.

\section{Experimental details}

\subsection{The ASK instrumentation}

The ASK instrument consists of three Andor iXon EMCCD detectors, each equipped with a Kowa lens, $75 \mathrm{~mm}$ F/1. The resulting field of view is $6.1^{\circ} \times 6.1^{\circ}$, corresponding to about $10 \times 10 \mathrm{~km}$ at an altitude of $100 \mathrm{~km}$. The spatial resolution at this height is $20 \mathrm{~m}$, and the temporal resolution is several frames per second. The instrument is pointed toward magnetic zenith. The aurora is imaged in three different passband filters simultaneously. The characteristics of the filters are shown in Table 1. The first filter, ASK1, isolates the two molecular oxygen bands $\mathrm{O}_{2}^{+} 1 \mathrm{~N}(1,0)$ and $\mathrm{O}_{2}^{+} 1 \mathrm{~N}(2,0)$ in the ionospheric E-region. The second filter, ASK2, is used to detect the forbidden doublet lines of $\mathrm{O}^{+}$in the F-region. This emission is produced by the $\mathrm{O}^{+}\left({ }^{2} \mathrm{P}-{ }^{2} \mathrm{D}\right)$ transition, with one stronger line at $7319 \AA$ and one weaker at $7330 \AA$. This wavelength region is contaminated by emission from $\mathrm{N}_{2} 1 \mathrm{PG}$ bands as well as $\mathrm{OH}$ airglow. The third filter, ASK3, isolates a line of atomic oxygen at $7774 \AA$. In the F-region, this line is caused by excitation of atomic oxygen $\left(\mathrm{O}+\mathrm{e}^{-}\right)$whereas in the E-region it results from dissociative excitation of molecular oxygen $\left(\mathrm{O}_{2}+\mathrm{e}^{-}\right)$. ASK was absolute intensity calibrated by using reference stars.

During the 2005/2006 campaign, ASK was deployed at the EISCAT Svalbard Radar (ESR) site $\left(78.15^{\circ} \mathrm{N}, 16.03^{\circ} \mathrm{E}\right)$. The cameras were pointed in the direction of magnetic zenith (azimuth $181.2^{\circ}$ east of north, elevation $81.6^{\circ}$ ). Measurements from other instruments located nearby provide an important contribution to studies with ASK. The first winter campaign was planned such that the Spectrographic Imaging Facility (SIF) would support the ASK measurements.

\subsection{The SIF instrumentation}

The Spectrographic Imaging Facility (SIF) is located $7 \mathrm{~km}$ from the ESR site, at the auroral station in Adventdalen (Sigernes et al., 2002). The platform consists of six instruments: the HiTIES (High Throughput Imaging Echelle Spectrograph), four narrow-band photometers and one narrowfield intensified video camera (McWhirter et al., 2001). The HiTIES imaging spectrograph is designed to measure spectral characteristics from several wavelength intervals simultaneously with high resolution. Emissions from the aurora pass through the slit corresponding to eight degrees on the sky, before the light is dispersed by an echelle grating. In the intermediate image plane a filter mosaic is placed (Fig. 1), to select non-contiguous wavelength intervals of interest (Chakrabarti et al., 2001). The mosaic filter was specially designed to coordinate with ASK by including the same spectral features. The mosaic consists of four spectral filters. Panel $\mathrm{O}_{2}^{+}(5620)$, covers the wavelength interval 5580-5650 ̊ to study $\mathrm{O}_{2}^{+}$emission, which contains the spectral range of ASK1. Panel O ${ }^{+}$(7319) (7280-7400 ̊), includes the same spectral features as ASK2. Panel OI(8446) is centered near an emission line from atomic oxygen, to be compared with the atomic oxygen line at $7774 \AA$ as measured by ASK3. Panel $\mathrm{H}_{\beta}(4861)(4800-4900 \AA)$ is designed to detect $\mathrm{H}_{\beta}$ emission from proton precipitation. HiTIES, with its high spectral resolution of $1 \AA$, provides important information about the spectral surroundings of the auroral emission detected by the ASK cameras. The SIF narrow-field video camera is an ICCD imager with a $<6450 \AA$ cut-off filter. It has a field of view of $16^{\circ}$ by $12^{\circ}$. The cut-off filter is selected to eliminate long-lived emissions at shorter wavelengths.

\section{Results}

Three events have been chosen to analyse different auroral structures for cases of low and high energy aurora, and a mixture of both. In the first event low energy precipitation was detected as an unstructured auroral arc passed slowly through the ASK field of view. In the second event the precipitating 
Table 1. The characteristics of the three passband filters in ASK.

\begin{tabular}{lllll}
\hline & Central $\lambda$ & FWHM & species & altitude \\
\hline ASK 1 & $5620 \AA$ & $26 \AA$ & $\mathrm{O}_{2}^{+} 1 \mathrm{~N}$-bands & E-region \\
ASK 2 & $7319 \AA$ & $10 \AA$ & $\begin{array}{l}\mathrm{O}^{+}+\text {contaminants } \\
\left(\mathrm{OH} \text { Airglow }+\mathrm{N}_{2} 1 \mathrm{PG} \text { bands }\right)\end{array}$ & $\begin{array}{l}\text { F-region } \\
\text { (E- \& F-region) }\end{array}$ \\
ASK 3 & $7774 \AA$ & $15 \AA$ & $\begin{array}{l}\mathrm{O}\left(\mathrm{O}+\mathrm{e}^{-}\right) \\
\mathrm{O}\left(\mathrm{O}_{2}+\mathrm{e}^{-}\right)\end{array}$ & $\begin{array}{l}\text { F-region } \\
\text { E-region }\end{array}$ \\
\hline
\end{tabular}

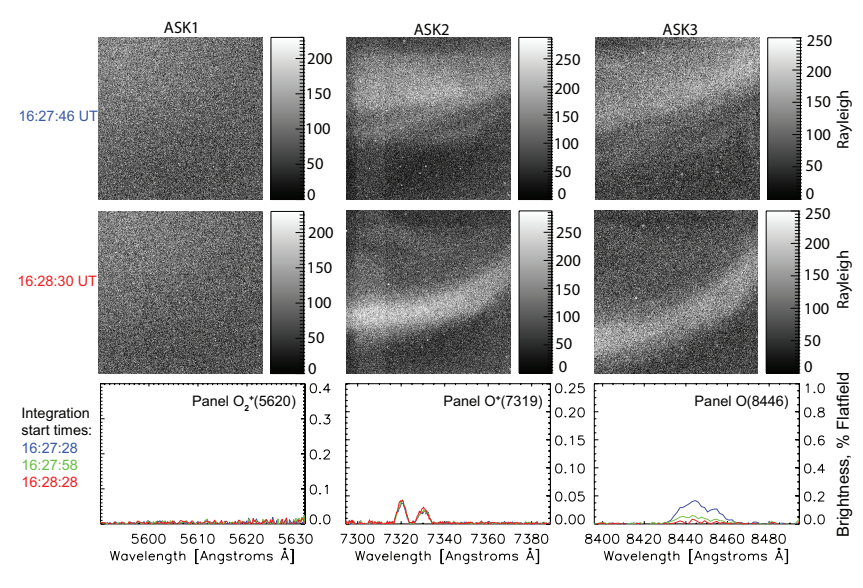

Fig. 2. ASK images and SIF line spectra from event 1, 2 January 2006, 16:00 UT. The top two rows of panels show ASK images from two different times during this event. The auroral arc seen in ASK2 and ASK3 is not detectable in ASK1, and the corresponding SIF line spectra (third row) confirm that the $\mathrm{O}_{2}^{+}$emission is at the noise level.

electrons were found to be more energetic. At this time dynamic, thin structures were observed in the aurora. The third event started and ended with low energy electron precipitation, with a period of high energy precipitation in between. Rays were seen in ASK at the beginning and the end of the event, but during the period of high energy precipitation the aurora became more dynamic, with rapid changes and narrow structures embedded in a broader arc. A more detailed description of the events is given below.

\subsection{Event 1, 2 January 2006, 16:00 UT - low energy aurora}

The top two rows of Fig. 2 show ASK images at 16:27:46 UT (top row) and 16:28:30 UT (second row), on 2 January 2006. During this one minute interval, a "quiet" arc was passing through the field of view of the ASK cameras. The arc is seen only in ASK2 and ASK3. Simultaneous spectra obtained with SIF are shown in Fig. 3; the bottom row of panels in Fig. 2 show the corresponding line spectra for three time intervals during the arc passage. The integration time for the SIF spectra is $30 \mathrm{~s}$, whereas the ASK images are recorded

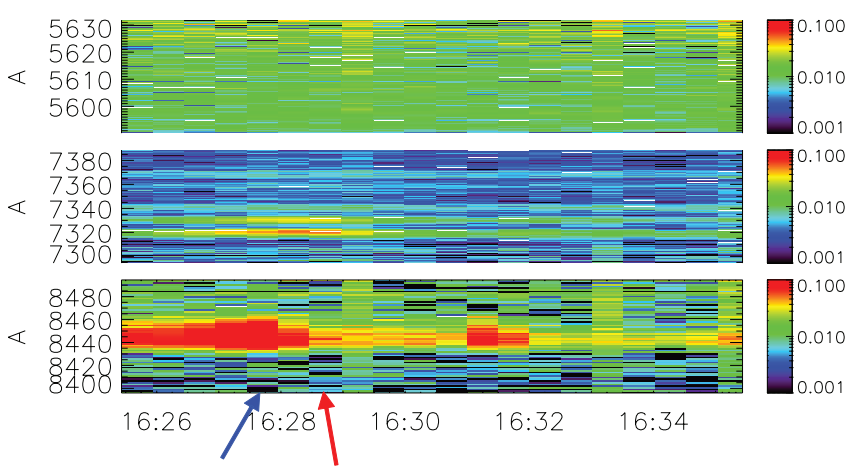

Fig. 3. SIF spectra for event 1 . The $\mathrm{O}_{2}^{+}(5620)$ panel (top panel), detects the same emission as ASK1, and is at the noise level. In the second and third panels (panel $\mathrm{O}^{+}(7319)$ and panel $\mathrm{OI}(8446)$ ) the oxygen emissions detected by ASK2 and ASK3 during the passage of the arc are seen. The arrows indicate the time intervals in which the ASK images in Fig. 2 were taken, with the same colour code.

with a time resolution of four frames per second. From ASK1 and the first panel of Fig. 3 it is evident that the molecular oxygen emissions are at the noise level. Thus, there are no E-region emissions. Instead the $\mathrm{O}^{+}$doublet dominates in the ASK2 passband, as can be seen from the HiTIES panel $\mathrm{O}^{+}(7319)$ and from the line spectrum. The $8846 \AA$ emission, from atomic oxygen in the near infrared, is detected in $\mathrm{Hi}-$ TIES panel OI(8446) and is seen in the rightmost line spectrum. This emission has similar excitation sources as the $\mathrm{F}$ region emission measured by ASK3 at $7774 \AA$. Assuming an emission altitude in ASK3 of around $200 \mathrm{~km}$, the arc can be estimated to have a width of about $3.5 \mathrm{~km}$ when viewed in the magnetic zenith. This is an example of aurora caused by low energy precipitating electrons.

\subsection{Event 2, 2 January 2006, 21:00 UT - high energy au- rora}

Shortly after 21:00 UT on the same day as event 1 very dynamic auroral emissions were detected, with many wavelike forms and folds. Figure 4 shows four panels of ASK images from this period, starting at 21:13:37 UT. The observed structures are almost identical in all three ASK cameras during 


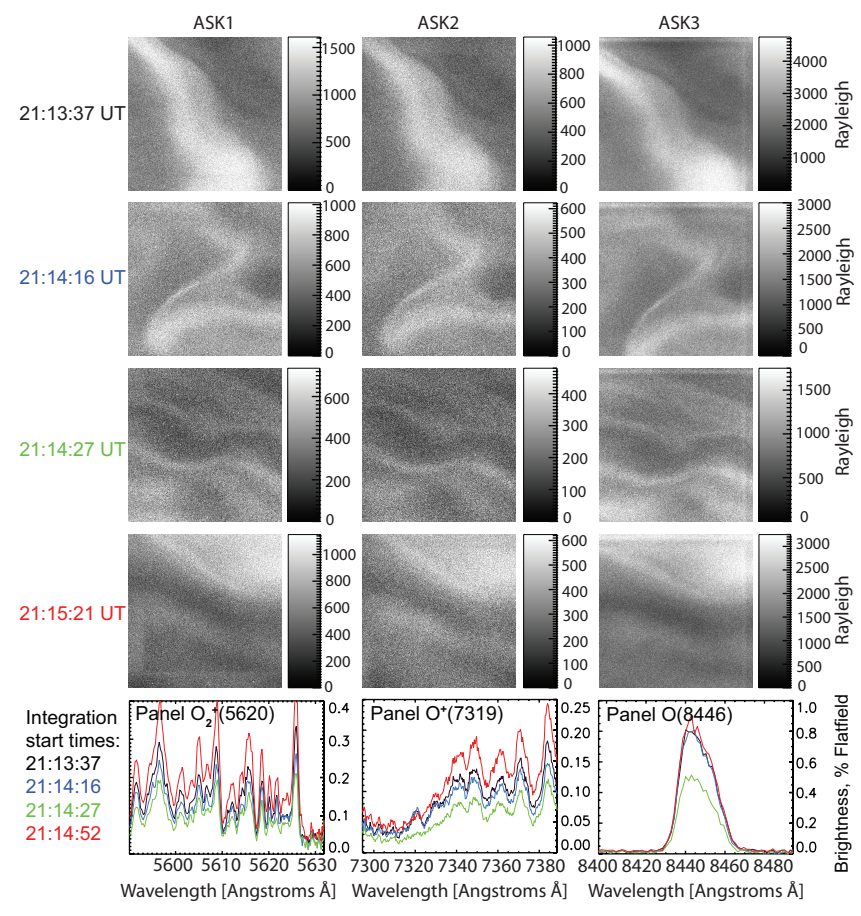

Fig. 4. ASK images for event 2, 2 January 2006, 21:00 UT. Rapid changes of the auroral forms are characteristic for this event, and the aurora is almost identical in all three ASK cameras. The bottom row of panels shows the SIF line spectra at the times of the ASK images. The scale of the line spectra is the same as in Fig. 2.

this event. The integration time for SIF is the same as for event 1 , namely $30 \mathrm{~s}$, and the bottom panels of the figure show the line spectra for four time intervals. The ASK images have been chosen to show a snapshot of the auroral structures from each SIF time interval. Panel $\mathrm{O}_{2}^{+}(5620)$ of the spectra (top panel in Fig. 5) and the corresponding line spectra now show strong $\mathrm{O}_{2}^{+} 1 \mathrm{~N}$ bands, as compared to the first event when only noise was detected in this wavelength region (note that the same intensity scale is used in the line spectra for different events). As time elapses the $\mathrm{N}_{2}$ bands in panel $\mathrm{O}^{+}(7319)$ become increasingly dominant over the $\mathrm{O}^{+}$doublet. This indicates that the emission detected in ASK2 originates from the contaminant $\mathrm{N}_{2}$ 1PG band in the E-region. In ASK3 the emissions are caused by the $\mathrm{O}_{2}+\mathrm{e}^{-}$ reaction in the E-region, since the SIF panel $\mathrm{O}_{2}^{+}(5620)$ shows strong $\mathrm{O}_{2}^{+} 1 \mathrm{~N}$ bands. Thus, all three cameras contain emissions originating in the E-region, confirming that this is a case of high energy electron precipitation. Very thin arc elements are observed in the dynamic structures during this event. By assuming that the detected E-region emission in ASK3 is at $120 \mathrm{~km}$ altitude, the widths of the most narrow structures are found to be less than $400 \mathrm{~m}$. When studying several ASK images during the fourth time interval it became evident that the small scale structures that were a character-

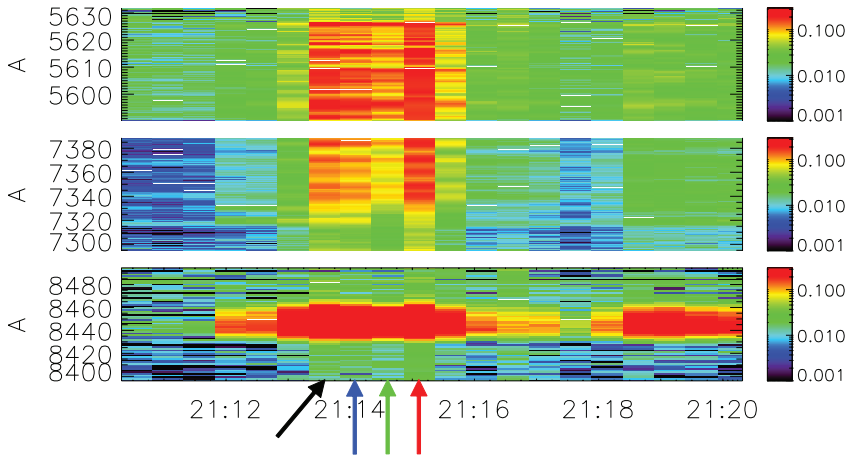

Fig. 5. SIF spectra for event 2. In panel $\mathrm{O}_{2}^{+}$(5620) (top panel) $\mathrm{O}_{2}^{+}$ $1 \mathrm{~N}$ bands excited in the E-region are seen. In panel $\left.\mathrm{O}^{+}(7319)\right)$, the oxygen doublet is overwhelmed by emission caused by the $\mathrm{N}_{2} 1 \mathrm{PG}$ band, which is a typical high energy aurora feature. The bottom panel (panel OI(8446)) shows the oxygen line emission during this event. The arrows mark the times of the ASK images in Fig. 4.

istic feature of this event were part of evolving larger scale structures.

\subsection{Event 3, 26 January 2006, 17:30 UT - mixture of low and high energy aurora}

Figure 6 shows images from the SIF ICCD imager with a $<6450 \AA$ A cut-off filter (left column of images), ASK images from ASK2 and ASK3 (middle and right column of images) and HiTIES spectra (bottom row), obtained at 17:30 UT on 26 January 2006. During this event ASK1 was not available. The SIF camera has a time resolution of 2.8 frames per second for this period. The position of the spectrograph slit is marked on all SIF camera images, with panel $\mathrm{O}^{+}(7319)$ and $\mathrm{O}_{2}^{+}(5620)$ coloured purple and panel $\mathrm{H}_{\beta}(4861)$ and $\mathrm{OI}$ (8446) coloured yellow. North direction is shown in the top row of images. The time resolution of the ASK cameras is 5 frames per second.

The ASK and SIF camera images show that this event initially contained rays, which within one minute evolved to dynamic thin arcs, and then back to rays again. In panel $\mathrm{O}^{+}(7319)$ of the HiTIES spectra for this event (second panel of Fig. 7) the oxygen doublet is enhanced and a burst of $\mathrm{N}_{2}$ bands can be seen for the two time intervals marked with blue and green in Fig. 6. It is during these time intervals, when high energy molecular emissions dominate, that thin arc elements with fluid-like motions are seen. During the time intervals marked with black and red, when the oxygen doublet and hence low energy emission dominates (see line spectra for panel $\mathrm{O}^{+}(7319)$ in Fig. 6), the aurora is characterised by slowly evolving rays. Consequently, rayed structure is here associated with the presence of low energy emission, which is in agreement with the results of Ivchenko et al. (2005). It is important to note, however, that the aurora is not evenly filling the HiTIES panels during the rayed periods. 


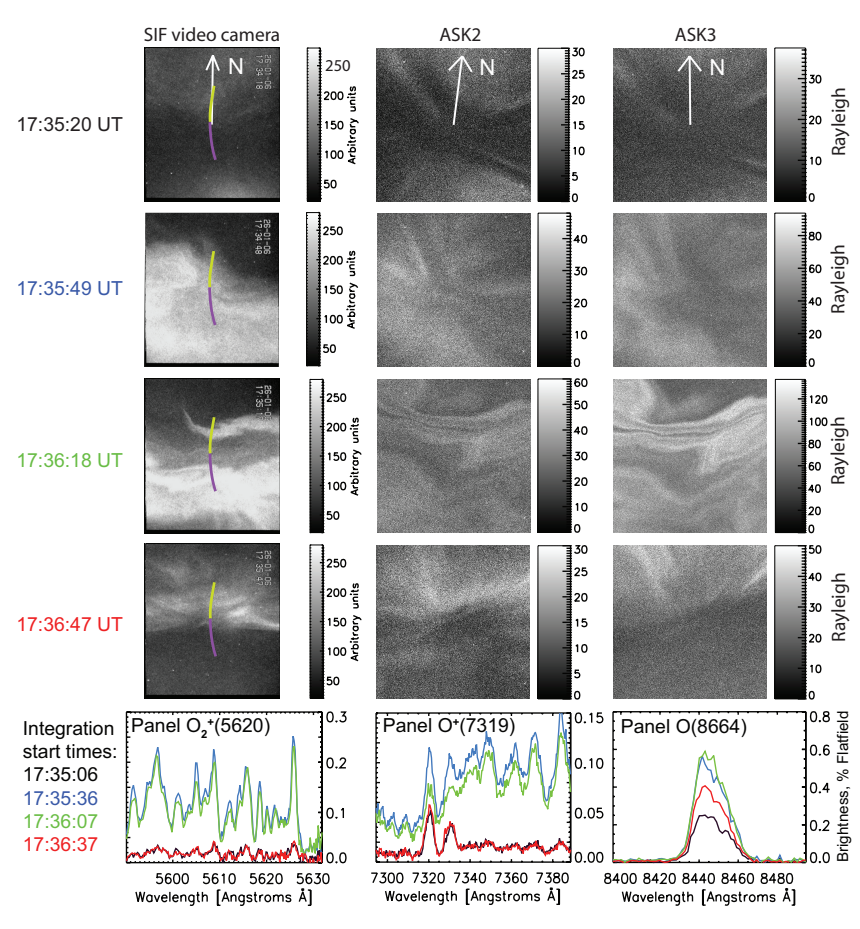

Fig. 6. ASK images, SIF video camera images and SIF line spectra for event 3, 26 January 2006, 17:30 UT. The first column of images shows the aurora during four different times, captured with the SIF ICCD imager. The second and third column of images show concurrent ASK2 and ASK3 images. The north direction is indicated in the top row of images. The bottom row of panels show the SIF line spectra.

During the two low energy time intervals (black and red), the brightest auroral elements are not in HiTIES field of view for panels $\mathrm{O}_{2}^{+}(5620)$ and $\mathrm{O}^{+}(7319)$. Comparing the two high energy time intervals (marked with blue and green), both in the ASK images and in panel $\mathrm{O}^{+}(7319)$ of the HiTIES spectra, it can be seen that more ray-like structures are visible within the system of arcs in the blue interval, when the oxygen doublet is still strong compared with the $\mathrm{N}_{2}$ bands. In the green period, the ASK images now show purely thin dynamic arc structures and HiTIES panel $\mathrm{O}^{+}(7319)$ shows that the $\mathrm{N}_{2}$ bands are dominating more compared with the weakening oxygen doublet. For these time periods the aurora is present on both sides of the HiTIES slit equally. The thinnest arc elements during this event are measured from the emissions in ASK3 (again assuming the altitude of the emissions to be $120 \mathrm{~km}$ ) and are found to be less than $200 \mathrm{~m}$ wide.

The difference in the field of view of ASK and the SIF imager is illustrated in Fig. 8, where the SIF image from 17:36:18 on 26 January is displayed in red, and the ASK3 image from the same time is overlaid in blue. The images are in the same orientation (with respect to the distant stars), so that objects at a finite distance exhibit parallax displacement between the two observation points. The displacement is par-

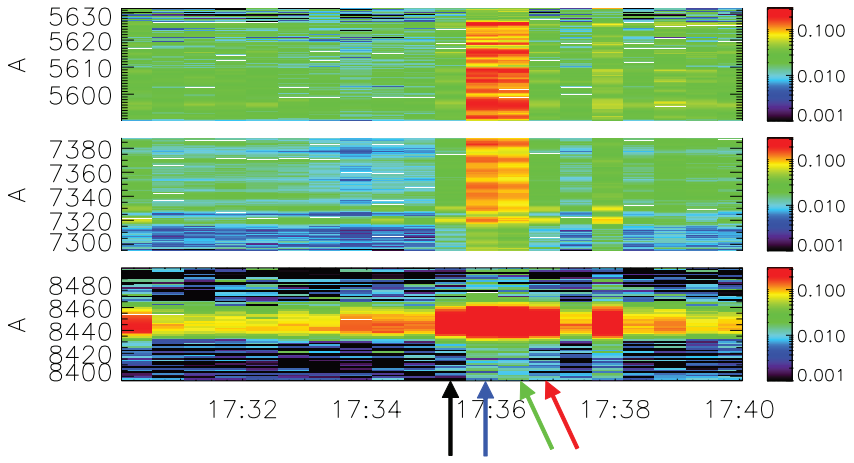

Fig. 7. SIF spectra for event 3. The molecular band emissions are seen during a time of high energy aurora at about 17:36 UT in panel $\mathrm{O}_{2}^{+}$(5620) (top panel) and panel $\mathrm{O}^{+}$(7319) (second panel). The bottom panel (panel OI(8446)) confirms the detection of atomic oxygen in ASK3 during this event. The arrows indicate the times of the ASK images seen in Fig. 6.

allel to the direction of the baseline between the ASK and SIF (vertical in the figure). The parallax between the edge of an auroral feature clearly identifiable in both cameras is marked with the white line in the figure. Since the shapes of the aurora are practically the same when viewed stereoscopically, they must come from a rather narrow altitude region (otherwise the features at large range would exhibit smaller parallax shift). From the scale plotted to the right, it is evident that the altitude of the emissions is about $100 \mathrm{~km}$. It should also be noted, that in this case the position and shape of the emissions in ASK2 and ASK3 cameras are about the same, indicating that they originate from the same altitude. This suggests that most of the features seen in ASK2 come from $\mathrm{N}_{2}$ contaminant emission, which is also strong in the HiTIES spectra (see Fig. 6).

\section{Intensity ratios}

By analysing the intensity ratio of the $\mathrm{O}_{2}^{+}$emissions (detected in ASK1) and the OI emissions detected in ASK3, $\mathrm{O}_{2}^{+} / \mathrm{OI}$, it is possible to extract information on the energy distribution and differences in particle flux during an auroral event, when assuming emission from the same altitude. Figure 9a shows images from all three channels, taken during the high energy event (event 2) discussed in Sect. 3.2. The auroral structures are similar in all three channels at the same instant of time. This means that the emissions originate in the E-region, as expected for high energy precipitation. If we study the ratio of the ASK1 and ASK3 images we see that the intensity ratio is not completely uniform (Fig. 9b). The bright auroral arc element stretching in the east-west direction and situated to the north of the field of view is still visible in the ratio image, as well as an area of intensification equatorward of the arc, to the left in the image. A darker region is situated 
Parallax from ASK and SIF at 17:36:18 on 26 January 2006

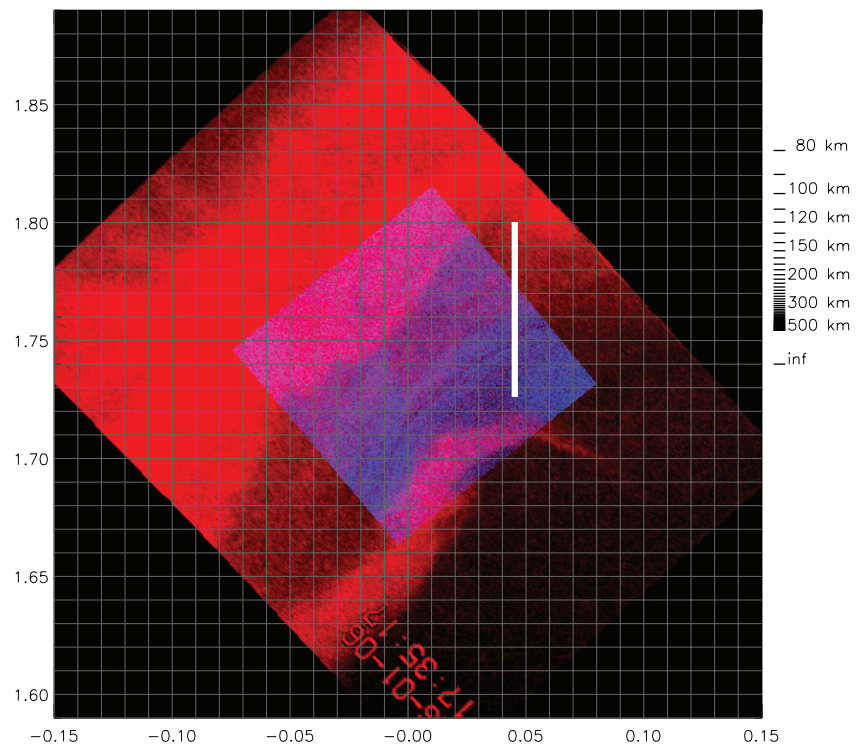

Fig. 8. ASK image (blue) plotted on top of SIF image (red), to illustrate the difference in field of view, and the parallax (white line) caused by the $7 \mathrm{~km}$ distance between the imagers. The grid step is 0.01 rad.

north of the filament. These three regions are marked with blue, green and red in 9c, respectively. A higher ratio means that the $\mathrm{O}_{2}^{+}$emission is relatively stronger than the OI emission in these structures. The reason for this is that the ratio of $\mathrm{O}_{2}$ and $\mathrm{O}$ concentration is rapidly decreasing with altitude, so that a higher $\mathrm{O}_{2}^{+} / \mathrm{OI}$ ratio indicates lower emission altitude, i.e. higher characteristic energy. The assumption of the same emission altitude for $\mathrm{O}_{2}^{+}$and $\mathrm{OI}$ may seem counter-intuitive, since one expects the low energy to excite OI primarily in the F-region, and $\mathrm{O}_{2}^{+}$should come from the E-region. However, both emissions originate at altitudes where energy deposition takes place, which is a relatively narrow altitude layer for high energy precipitation. The ratio of emissions is roughly determined by the ratio of the parent constituents $\left(\mathrm{O}_{2}\right.$ and $\left.\mathrm{O}\right)$. Of course, if mixed energy precipitation is present, the only accurate way of reconstructing the precipitation energy distribution would be to reconstruct the three dimensional distribution of both emissions from the image plane projections. Identical morphology of the aurora in $\mathrm{O}_{2}^{+}$and OI channels is a good indication of the applicability of the emission ratio analysis, whereas presence of features with clear altitude separation of emissions and perspective effects means that a more complicated analysis should be employed.

From the ratio image the three regions with different intensities can be discerned, and if we plot the ratio of $\mathrm{O}_{2}^{+}$and OI emissions versus the OI emission for each pixel in these regions (Fig. 9c), and mark the pixels from a specific region with the same colour as the region, we see these that each region has a different mean ratio.

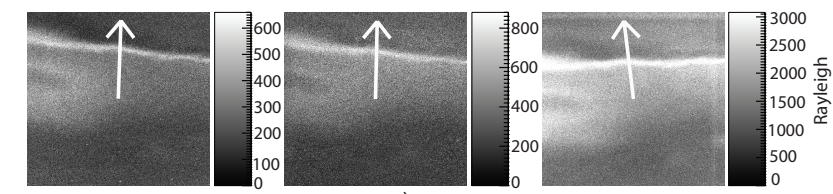

a)

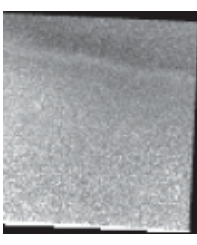

b)

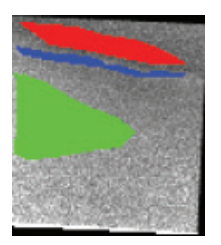

c)

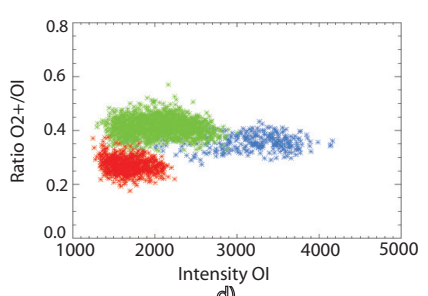

d)
Fig. 9. (a) Images from ASK1 $\left(\mathrm{O}_{2}^{+}\right)$, ASK2 $\left(\mathrm{O}^{+}\right)$and ASK3 (OI) at 21:14:02,750 on 2 January 2006 . The north direction in the images is indicated with an arrow. (b) $\mathrm{O}_{2}^{+} / \mathrm{OI}$ ratio plot. (c) ratio plot marked with three regions with different characteristic intensities (d) plot of ratio against OI emission for each pixel in the three regions. It is clear that the three regions have different ratios.

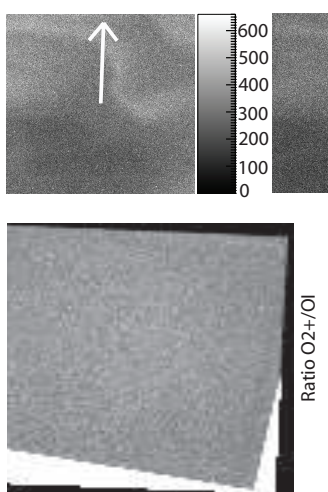

b)
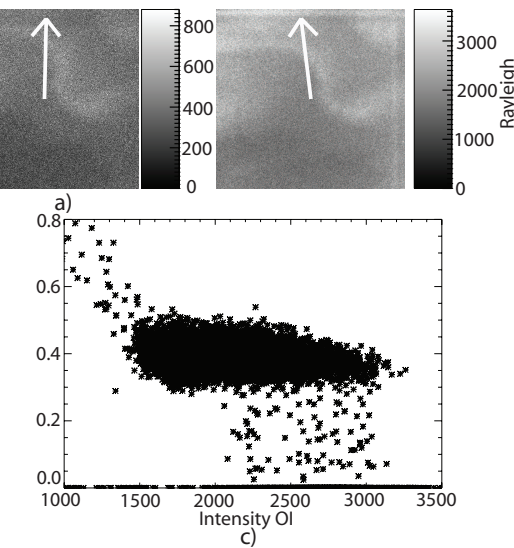

Fig. 10. Same format as Fig. 9, for images taken at 21:14:14,500 on 2 January 2006.

From the same event, Fig. 10a shows images taken at 21:14:14,500 UT. At this time a curl/fold is passing the field of view, and again the auroral features are detected in all three channels, indicating high energy aurora. The ratio of $\mathrm{O}_{2}^{+}$emission to OI emission is plotted in Fig. 10b. This time, the ratio image contains little structure and is almost completely smooth. This is also evident from the ratio plot in Fig. 10c, where there is now only one main ratio value for all the pixels. This means that the $\mathrm{O}_{2}^{+} / \mathrm{OI}$ emission ratio is close to constant over the image. Thus, the curl seen in the individual images cannot be the result of varying precipitation energy in that region, but must be the result of higher particle flux in the curl, compared to the surroundings. 


\section{Discussion}

Davis (1978) and Haerendel (1999) have both pointed out the importance of considering the imaging contrast of narrow filaments, since their apparent width will depend on this property of the imaging system. The brightness of thin structures in many cases is found to be only $10 \%$ above the background level. Figure 9 provides information on the contrast between the bright horizontal filament detected at 21:14:02,750 on 2 January 2006, and its background. The ratio plot shows that the intensity of the OI emission varies between approximately $1300 \mathrm{R}$ and $4000 \mathrm{R}$ in this image. The amplitude of the brightest parts of the filament is thus $200 \%$ that of the lowest intensity of the aurora. The same comparison can be made for the intensity of the OI emission in Fig. 10, taken at $21: 14: 14,500$ on 2 January 2006 . The intensity of OI varies here between $1500 \mathrm{R}$ and $3300 \mathrm{R}$, which gives a contrast of up to $120 \%$. Lanchester et al. (1997) also discuss the importance of contrast when studying auroral images. In their event, also discussed by Haerendel (1999), an arc in the camera field of view appeared as a narrow filament of $100 \mathrm{~m}$ width, only when the exposure time per frame was reduced to as short as $2.5 \mathrm{~ms}$. At longer exposures, the arc appeared as a wide and saturated feature. The exposure time of ASK in the events described here is $0.2 \mathrm{~s}$, so that a narrow arc element moving rapidly across the field of view will be smeared out in the image during this time.

For the high energy event, the $\mathrm{O}_{2}^{+} / \mathrm{OI}$ intensity ratio was studied. If the ratio is not uniform this means that these regions contain precipitation of electrons with higher energies than the regions nearby, since an enhancement of $\mathrm{O}_{2}^{+}$is a signature of high energy. This is what is observed in the first ratio image; the visible arc element in the three ASK channels is the result of higher energy precipitation compared to the surroundings. About $12 \mathrm{~s}$ later a fold is seen in the ASK channels, but this time the $\mathrm{O}_{2}^{+} / \mathrm{OI}$ intensity ratio is smooth. The brighter fold must be primarily a result of higher particle flux, rather than higher energy. Combining the ratio with modelling of emissions will allow a quantitative determination of the characteristic energy. A full analysis of this kind is beyond the scope of the work presented here, but will be a subject for future work.

Borovsky (1993) presented a number of theories to account for the production of auroral arcs with widths of the order of km, but smaller structures still lack a complete understanding. Optical and modelling work (Lanchester et al., 1997, 2001) indicates that there are many different scale sizes operating in the lower magnetosphere, often producing narrow intense features within a larger "arc" region. The broader features can be explained by the theories above, and in the case of arcs with a width of the order $10 \mathrm{~km}$ the energies are consistent with an acceleration mechanism that could produce the commonly observed inverted- $\mathrm{V}$ distributions in energy. The very small scale structures requiring a full theoretical explanation fall in the range of typical auroral widths found by Maggs and Davis (1968). Trondsen and Cogger (2001) used the narrow-field Portable Auroral Imager (PAI) to observe auroral structures with thicknesses of less than $120 \mathrm{~m}$. ASK has a similar spatial resolution as PAI $(20 \mathrm{~m}$ compared with $10 \mathrm{~m}$ for PAI). In the three events studied here the narrowest width is $\sim 200 \mathrm{~m}$.

\section{Conclusions}

ASK is a newly developed high resolution optical instrument to observe small scale auroral features in several emission lines simultaneously. We report on the first results from this imager, where auroral morphology has been analysed based on the relative changes in the characteristic energy of the precipitating electrons. Three events from the first campaign were chosen, with different energy signatures. Emission spectra from the SIF spectrograph were also studied in conjunction with ASK operations to provide spectral information on the source of the auroral emissions.

These events suggest that the aurora is much less dynamic during low energy precipitation than during events with high energy precipitation. Rays are primarily seen during events with a mixture of low and high energy precipitation, whereas rapid, thin filaments are signatures of high energy. This observation will be explored further in a following paper. The widths of the auroral structures are also of different scale sizes in the two cases. In the low energy event the arc that is slowly drifting through the field of view has a width of about $3.5 \mathrm{~km}$, whereas arc elements during the two high energy events show widths of less than $400 \mathrm{~m}$ and $200 \mathrm{~m}$, respectively. A more extensive survey of auroral widths is planned with the ASK data, with the aim of understanding the processes that produce these dynamic and filamentary structures.

Acknowledgements. During this work H. Dahlgren spent 2 months working in Southampton (summer 2006) by support from the Royal Astronomical Society. N. Ivchenko is supported by the Swedish Research Council. Work at the Royal Institute of Technology was partially supported by the Swedish National Space Board and the Alfvén Laboratory Centre for Space and Fusion Plasma Physics. J. Sullivan was supported by, and the ASK instrument was funded by the PPARC of the UK.

Topical Editor U.-P. Hoppe thanks A. Aikio and another referee for their help in evaluating this paper.

\section{References}

Borovsky, J. E.: Auroral arc thicknesses as predicted by various theories, J. Geophys. Res., 98, 6101-6138, 1993.

Chakrabarti, S., Pallamraju, D., Baumgardner, J., and Vaillancourt, J.: HiTIES: A High Throughput Imaging Echelle Spectrograph for ground-based visible airglow and auroral studies, J. Geophys. Res., 106, 30337-30 348, 2001

Davis, T. N.: Observed characteristics of auroral forms, Space Sci. Rev., 22, 77-113, 1978. 
Haerendel, G.: Origin and dynamics of thin auroral arcs, Adv. Space Res., 23, 1637-1645, 1999.

Hallinan, T. J., Kimball, J., Stenbaek-Nielson, C., H., Lynch, K., Arnoldy, R., Bonnell, J., and Kintner, P.: Relation between optical emissions, particles, electric-fields and Alfvén waves in a multiple rayed arc, J. Geophys. Res., 106, 15 445-15 454, 2001.

Ivchenko, N., Rees, M. H., Lanchester, B. S., Lummerzheim, D., Galand, M., Throp, K., and Furniss, I.: Observation of O+ (4P4D0) lines in electron aurora over Svalbard, Ann. Geophys., 22, 2805-2817, 2004,

http://www.ann-geophys.net/22/2805/2004/.

Ivchenko, N., Blixt, E. M., and Lanchester, B. S.: Multispectral observations of auroral rays and curls, Geophys. Res. Lett., 32, L18 106, doi:10.1029/2005GL022650, 2005.

Kaila, K. U.: Determination of the energy of auroral electrons by the measurements of the emission ratio and altitude of aurorae, Planet. Space Sci., 37, 341-349, 1989.

Lanchester, B. S., Rees, M. H., Lummerzheim, D., Otto, A., Frey, H. U., and Kaila, K. U.: Large fluxes of auroral electrons in filaments of 100 m width, J. Geophys. Res., 102, 9741-9748, 1997.

Lanchester, B. S., Rees, M. H., Lummerzheim, D., Otto, A., Sedgemore-Schulthess, K. J. F., Zhu, H., and McCrea, I. W.: Ohmic heating as evidence for strong field-aligned currents in filamentary aurora, J. Geophys. Res., 106, 1785-1794, 2001.

Maggs, J. E. and Davis, T. N.: Measurements of the thicknesses of auroral structures, Planet. Space Sci., 16, 205-209, 1968.

McWhirter, I., Furniss, I., Lanchester, B. S., Robertson, S. C., Baumgardner, J., and Mendillo, M.: A new spectrograph platform for auroral studies on Svalbard, in: Proceedings of Atmospheric Studies by Optical Methods, pp. 1-4, 2001.
Otto, A. and Birk, G. T.: Formation of thin auroral arcs by current striation, Geophys. Res. Lett., 20, 2833-2836, 1993.

Paschmann, G., Haaland, S., and Treumann, R.: Auroral Plasma Physics, Springer, 2003.

Semeter, J.: Critical comparison of OII(732-733 nm), OI(630 nm), and N_2(1PG) emissions in auroral rays, Geophys. Res. Lett., 30, 1255, doi:10.1029/2002GL015828, 2003.

Semeter, J. and Blixt, E. M.: Evidence for Alfvén wave dispersion identified in high-resolution auroral imagery, Geophys. Res. Lett., 33, 13 106, doi:10.1029/2006GL026274, 2006.

Semeter, J., Lummerzheim, D., and Haerendel, G.: Simultaneous multispectral imaging of the discrete aurora, J. Atmos. SolarTerr. Phys., 63, 1981-1992, 2001.

Sigernes, F., Svenøe, T., and Deehr, C. S.: The Auroral Station in Adventdalen, Svalbard $\left(78^{\circ} \mathrm{N}, 15^{\circ} \mathrm{E}\right)$, Chinese Journal of Polar Science, 13, 67-74, 2002.

Stasiewicz, K., Bellan, P., Chaston, C., Kletzing, C., Lysak, R., Maggs, J., Pokhotelov, O., Seyler, C., Shukla, P., Stenflo, L., Streltsov, A., and Wahlund, J.-E.: Small scale Aflvénic structure in the aurora, Space Sci. Rev., 92, 423-533, 2000.

Trondsen, T. S. and Cogger, L. L.: Fine-scale optical observations of aurora, Phys. Chem. Earth, 26, 179-188, 2001.

Vogt, J., Frey, H. U., Haerendel, G., Höfner, H., and Semeter, J. L.: Shear velocity profiles associated with auroral curls, J. Geophys. Res., 1999.

Wagner, J. S., Sydora, R. D., Tajima, T., Hallinan, T., Lee, L. C., and Akasofu, S.-I.: Small scale auroral arc deformations, J. Geophys. Res., 88, 8013-8019, 1983. 